\title{
¿Qué hay detrás de la profesión de educar? Un amor a la vida
}

\author{
Tany Giselle Fernández Guayana* / fernandez@unisabana.edu.co \\ Francisco Javier Bernal Sarmiento** / franciscobernal@loscerros.edu.co
}

\section{Resumen}

El presente artículo es una reflexión en torno al significado de la profesión de educar y busca generar conciencia en los futuros educadores sobre la relevancia de la carrera. Como sustento, realiza una descripción y una reflexión crítica sobre las habilidades y virtudes humanas que posibilitan al educador ser más que un profesor. Se concluye que la profesión cobra sentido cuando el docente realiza su quehacer pedagógico a partir de la entrega incondicional a sus estudiantes. La educación se convierte en una forma de felicidad que no modifica al mundo exterior, pero trasciende la calidad de la vida propia del educador y la de los educandos.

\section{Palabras clave}

Educar, virtudes, persona, entrega, felicidad.

\section{Introducción}

La educación, entendida como acción de crear e instruir de fuera hacia dentro (educare) y acto de guiar o conducir al ser humano (educere) (Casanova, 1991), requiere de la adecuada didáctica para lograrse, sin embargo, no es suficiente, hace falta "la pasión por enseñar". De acuerdo con las investigaciones realizadas por Day (2006), a pesar de las exigencias a las que estén expuestos los profesores, el amor por educar es el motor de toda labor pedagógica, porque cuando se ama lo que se hace, se logran superar los obstáculos, contagiando de vitalidad la propia existencia y la de los demás.

¿Cuánto hemos dedicado para terminar la carrera de licenciatura?, no sería coherente que después de tanto sacrificio se ejerza la labor por un "porque sí" o por tener un "puesto bien pago". Para los educadores se nos hace imprescindible tener una relación afectiva con la profesión. Los profesores no somos recreadores, somos educadores que encontramos el sentido de nuestra propia existencia al entregarnos. A través de esa dadivosidad posibilitamos que los educandos, sus padres, nuestros compañeros de trabajo y la sociedad se potencien buscando su mejora continua.

\footnotetext{
* Profesora en formación e investigadora. Licenciada en Pedagogía Infantil. Estudiante de la Especialización en Desarrollo Personal y Familiar. Instituto de La Familia, Universidad de La Sabana.

** Profesor-investigador. Magister en Educación. Facultad de Educación, Universidad de La Sabana.
} 
Con los cambios que ha tenido la sociedad moderna se ha ido modificando también el significado de la profesión de educar, ahora, se le ha considerado como la rama que se dedica únicamente a la enseñanza, empero va más allá. La educación es de la persona (docente) por las personas (educandos) y, a partir de esa entrega, el docente logra darle sentido a su quehacer $y$, en consecuencia, a su propia existencia. Es así como se afirma que el "amor a la vida se consigue con el paso del yoísmo a la filantropía".

Desde esa perspectiva, la consolidación de este artículo radica en generar conciencia a futuros educadores sobre la relevancia de la profesión y la influencia que tiene el desarrollo personal en la en la misma, porque educar trae consigo el amor, la entrega y la pasión, las bases que construyen esa felicidad tan anhelada.

\section{Para saber lo que debemos hacer, se \\ debe hacer lo que queremos saber}

La vida cobra sentido cuando se hace aquello que se quiere saber (Llano, 2002). La vida no tiene rumbo cuando se realizan acciones sin fin alguno, por eso, en la cotidianeidad, se recomienda articular un conocimiento práctico, con los conocimientos y creencias que cada persona defiende (arte regio), así, entonces, se sabrá vivir bien (Platón, citado por Llano, 2002, p.132); en pocas palabras, la vida cobra sentido cuando se actúa coherentemente.

Por parte, otra rama filosófica define la vida como un "arte" (Despeyroux, 2013, p.10), debido a que requiere de técnicas y saberes para seguir su curso. De acuerdo con Lorda (2006), así como "un pintor no puede pintar conociendo únicamente la teoría de los colores o haciendo trazos en un lienzo" (p.131), de igual forma sucede con el ser humano: no puede limitarse en la acumulación de conocimientos, también debe actuar con el fin de potenciar el saber ya adquirido. Mayor coherencia entre técnica y saber significa una mayor experiencia, ella posibilita que la persona actúe correctamente en determinadas situaciones y se vuelva "competente" (Lorda, 2006, p.19).
Pero, ¿qué sentido tiene el hacer de la vida un arte?, la respuesta es sencilla: para saber vivir, acto que conlleva a la felicidad a pesar de las vicisitudes, se debe aclarar que la vida no está predeterminada por una cuestión de suerte, no es como algunos pretenden: "unos nacen con estrella y otros nacen estrellados" (Bauman, 2011, p.8). Un hecho como lograr la vida no puede depender del azar, tampoco de la herencia, porque la existencia es inmanente y propia de cada ser, cada quien se labra esa felicidad a través de la coherencia entre actos y saberes.

En consecuencia, no es lo mismo que los profesores trabajemos por cumplir unos requisitos institucionales/gubernamentales, a que trabajemos por pasión, haciendo lo que amamos y amando lo que hacemos. Es verdad que en la actualidad enfrentamos una serie de imperativos externos, como trabajar en equipo, promover la cohesión social o dar ejemplo, entre otros (Day, 2006); sin embargo, esas exigencias no deben alejarnos de la coherencia entre nuestros principios y la práctica, de ser así, cabría la posibilidad de desistir de esta profesión, porque la vida misma se tornaría vana y efímera:

"En un programa titulado "ejecutividad" los docentes y la institución planificaron de cara al progreso de los estudiantes y los supervisaron de forma sistemática. Implementaron acciones generales, burocráticas y generalistas que provocaron que muchos profesores perdieran la pasión por educar con la que ingresaron a la profesión; el espacio para la espontaneidad, creatividad y para atender las necesidades imprevistas del aprendizaje de los niños se había contraído a medida que los profesores se enfocaban en alcanzar y cumplir los objetivos señalados por el gobierno" (Lyotard, 1979, p.30).

La entrega de un yo apasionado a la enseñanza de cada día, cada semana y cada curso escolar es una perspectiva que va más allá de enseñar. De acuerdo con Day (2006): "El hecho de tener una buena idea de qué hacer con los niños es solo el principio del trabajo de la educación" (2006, p. 34); razón por la cual se integra lo personal con lo profesional y la mente con la emoción. Lamentablemente, el trabajo docente está regido por nuestras cabezas y manos y no por el equilibrio entre 
sentimiento y pensamiento. En las últimas investigaciones (Ramsay, 1993; Macconi, 1993, White y Roesch, 1993; Lander, 1993; Altet, 1993), se ha detectado que los profesores que realizan su labor con fervor producen en los estudiantes resultados positivos, especialmente en la apertura al aprendizaje, las relaciones sociales y la aplicación de los conocimientos.

Dado lo anterior, la educación adquiere mayor significado cuando se relacionan las acciones con los conocimientos. Para saber vivir se hace imprescindible que los educadores amemos lo que hacemos y hagamos nuestra labor para saberla amar, porque nuestro quehacer cobra sentido cuando hacemos lo que queremos saber.

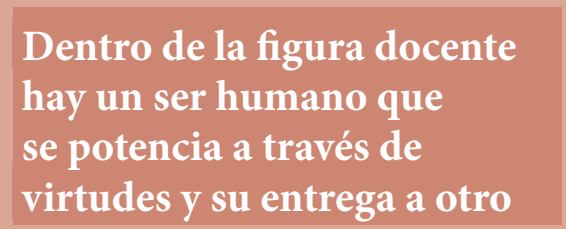

El hombre no es un ser acabado, no es perfecto y por consiguiente no está cumplido: "Su vida no se puede describir, hay que narrarla", expresa Llano (2002, p.28), y para hacerlo se requiere de tiempo y entrega. El tiempo contribuye a que la experiencia trascienda la naturaleza humana; la entrega posibilita el coexistir. De manera que la profesión de educar cobra sentido cuando hay tiempo para vivir y tiempo para donar la vida o "servir" (Hansen, 1999) a los educandos.
La identidad narrativa de la persona requiere de tiempo para que se despliegue: "un curso dramático hacia la plenitud, la perfección de la actividad en la que la vida esencialmente consiste" (Llano, 2002, p. 121). Hay que utilizar bien el tiempo, ya que hay actividades que tienen influencias positivas y negativas. Según Llano, el tiempo utilizado en la incorporación de hábitos buenos, posibilita el potenciamiento de la persona. Por ejemplo, cuando se dice la verdad se está portando como buen sujeto y a la vez se está "gastando bien el tiempo", porque se crece y se hace crecer a los demás (p. 31), de manera que no todo lo que hace el hombre sirve para abrir el camino a su vida lograda (como quiere ser), éste depende de los resultados de sus acciones. Vivir bien es mucho más que cubrir las necesidades primarias, consiste en lograr el bien humano y rectificar enseguida si se comete un error en contra del propio modo de ser (p. 32).

En efecto, mientras los profesionales de la educación gastemos el tiempo realizando acciones acordes con nuestra naturaleza, podremos entonces acrecentar esas virtudes que serán necesarias para lograr una mayor intensidad humana. El hecho no consiste en divinizarnos por ser virtuosos, pues los adultos también seguimos aprendiendo (Urbieta, 2006). Los profesores no somos únicamente una figura de autoridad que induce conocimientos a una tabula rasa, también somos humanos con defectos y virtudes que necesitamos potenciarnos mientras nos donamos a los estudiantes.
La entrega posibilita que los individuos crezcan y enriquezcan recíprocamente su naturaleza propia; gracias a la apertura de la intimidad (Cuadrado, 2010) se comparte lo que es cada persona (Kriedergan, citado por Llano, 2002; Stork y Aranguren, 2003). Los seres humanos son seres coexistentes que alcanzan el culmen de la vida (y paradójicamente su libertad) cuando hacen un vaciamiento del yo abriéndose amorosamente al otro, porque se comprende que lo que se tiene no es propio, sino de quien se ama (Stork y Aranguren, 2003). Es como el amor a Dios, a pesar de que el hombre sea un ávido conocedor de su presencia, le ama sobre todas las cosas porque se apunta hacia su ser mismo.

En éste sentido, la profesión de educar va más allá del simple hecho de enseñar, porque los docentes también crecemos y encontramos la felicidad cuando nos tejemos con la existencia de los estudiantes, como lo manifiesta Antonio Machado: "moneda que está en la mano, tal vez se deba guardar. La monedita del alma se pierde si no se da" (Llano, 2002, p.169). El quehacer pedagógico necesita de tiempo, tiempo para enseñar, aprender $y$ darse. No basta con pensar en sí, el amor no es un recipiente del que se va sacando hasta quedarse vacío, amarse y cuidar de sí implica amar y cuidar a los demás como a sí mismo: "es un camino digno de la vida que se nos regala y comparte" (Urbieta, 2006, p.82). 


\section{La identidad de la profesión de educar}

Actualmente, los profesores estamos expuestos a las exigencias del medio, como atender la indisciplina, la multiculturalidad, la falta de apoyo de algunos padres, las consecuencias de divorcios, la tecnología, los jefes, compañeros, estudiantes y padres, a todo esto de una manera diferente para cada uno y, por si fuera poco, atender los embates de la vida (Day, 2006; Isaacs, 2008). Con todos estos requerimientos, nuestra auténtica identidad oscila entre recreadores, cuidadores y hasta "madres". La verdad es que de algún modo sí ejercemos algo de esos roles, sin embargo, sólo la profesión de educar tiene a su cargo la: "enseñanza con fines formativos de diversas índoles" (Diker, 1997, p. 94).

Si bien los docentes tenemos a nuestra merced una gran variedad de funciones, ninguna de ellas es excusa para no ejercer diligentemente nuestro quehacer. No podemos comprometernos con un solo campo (teoría o práctica) porque estaríamos cosificando a la persona. Por el contrario, si entrelazamos acciones y saberes, lo personal y el posicionamiento ético, y el quehacer en relación a unos valores (Diker, 1997), la existencia cobra sentido. Los docentes debemos saber el arte, la técnica y el saber hacer, (Llano, 2002).

La relación entre teoría y práctica no es suficiente en la profesión de educar. De acuerdo con Isaacs (2008), los docentes requerimos de alcanzar las siguientes virtudes esenciales: a) Justicia, ayudar a las personas a descubrir el bien y a vivir bien para los demás, supone unos derechos y deberes que tienen que ser atendidos; b) Comprensión, concientización de que cada estudiante es diferente y tiene unas particularidades que influyen en su desempeño, por tal razón se exige y se actúa de acuerdo a esas condiciones y realidades; c) Optimismo, confiar razonablemente en las posibilidades y ayuda que se pueden recibir, distinguiendo lo que es aprovechable para sobrepasar los obstáculos con alegría.

Adicionalmente, Day (2006) menciona que los profesores debemos potenciar unas dimensiones que nos son particulares: a) Compromiso, dedicación intensiva que el educador tiene hasta el punto de sentir la necesidad de prestar atención a la propia formación continua; b) Continuo aprendizaje, adaptación y avance en circunstancias cambiantes para aprender nuevas tendencias en la educación; c) Apoyo mutuo, colaboración material y emocional para trabajar con otros; d) Eficacia, capacidad de mejorar la vida de los estudiantes a través de la práctica y el currículo oculto; e) Reflexión crítica, analizar el propio ejercicio profesional en busca de una mejora para la próxima intervención.

Dado lo anterior, se puede concluir que la identidad de la profesión de educar es enriquecida cuando se relaciona el ser con los saberes y las acciones que se realizan. Así entonces, el profesor puede actuar prudente y coherentemente frente a una realidad específica. Gracias a ello, puede desenvolverse eficazmente en la vida escolar poniendo en práctica sus virtudes: el compromiso, la entrega, el optimismo, la justicia, la reflexión, la comprensión y el aprendizaje continuo, para luego entregarse fielmente a sus estudiantes, carácter particular que da sentido a su profesión, su propia vida.

\section{El fin de la educación: la felicidad}

Se ha visto cómo la profesión de educar cobra sentido cuando hacemos lo que queremos saber, cuando nos entregamos a otros, cuando relacionamos la teoría con la práctica y cuando somos justos, comprensivos y optimistas, pero ¿será suficiente?, la respuesta es negativa. Todas las cosas que los docentes hacemos implican un esfuerzo, es así como se les valora y ama y como se logra ser plenamente feliz.

A la felicidad se le considera un conjunto de sensaciones agradables que buscan el gozo y evitan el dolor (Llano, 2002), pero quienes se centran en lo "que les gusta" están cayendo en un "relativismo moral" (Platón, citado por Llano p. 68), donde gobierna el libertinaje y las paraciencias. En tal sentido, se hacen las cosas en cuanto motivan, sin embargo, el placer no es un fin en sí mismo, no hay satisfacción con apariencias, habitualmente interesa la verdadera esencia de las cosas (p. 186). 
Entonces, se podría concluir que la felicidad se consigue cuando se alternan placeres y dolores, porque no todo lo que es bueno produce placer y a veces el sufrimiento o los sacrificios brindan recompensas más gratas. La idea del dolor consiste en vivirlo tal como es en vez de evitarlo, porque de tantos esfuerzos acordes con la naturaleza humana, la vida produce resultados positivos que hacen apreciar lo que se ha conseguido. Es "esa pasión que hace que lo que se haga le dé importancia a la vida, porque la felicidad se consigue cuando se busca cómo afrontar los sucesos vida" (Llano, 2002, p. 66).

Desde el campo educativo, se puede decir entonces que los educadores debemos sufrir por amor a nuestra profesión: "El profesor puede desarrollar una serie de tareas de enseñanza o de capacitación sin amar a los estudiantes. Mas si se preocupa por el auténtico bien de ellos, sufrirá, pero estará educando y tendrá mayores posibilidades de ser feliz en la vida" (Isaacs, 2008, p. 15). Porque el amor, de acuerdo con Llano (2002): "no es la marginación de la verdad, por el contrario, es la fuente de todo saber y la íntima energía que alimenta a cualquier comunidad humana" (p. 168). Por tanto, cuando los profesores nos involucramos en las situaciones de los educandos, nos afligimos y hacemos lo que esté a nuestro alcance para que logren afrontar el problema, y es allí cuando sen- timos una satisfacción inmensa por lo realizado, cuando sentimos la verdadera felicidad.

Hoy día hay maestros que están moralmente de capa caída y sufren del "síndrome del quemado" (Day, 2006, p. 88), por la creciente demanda de contratos de corta duración que provocan un grado de satisfacción mínima. De acuerdo con Evans (1997), se distinguen dos factores que contribuyen a la satisfacción profesoral: comodidad en el trabajo y realización en el trabajo. No obstante, la felicidad no es abastecida, se hace primordial implicar el acto educativo con lo emocional porque, a pesar de que los profesores suframos ante situaciones que viven los educandos, al involucrarnos, por amor a su ser, logramos darle sentido a la vida.

La felicidad entonces compromete a amar incondicionalmente, puesto que: "una cosa es tener pasión por la enseñanza y otra muy distinta ser un docente apasionado en todo tipo de condiciones de trabajo" (Day, 2006, p. 190). Se trata de recuperar la satisfacción que provoca el compartir con los estudiantes cada día, buscando: "el bien del otro en cuanto al otro" (Melendo, 2002, p. 17), afrontando los avatares para que los estudiantes sean felices. En pocas palabras, la felicidad la conseguimos cuando amamos nuestro quehacer sin importar las dificultades y viviendo la magia que trae consigo la labor de educar.
Conclusión

Es así entonces como se afirma que: el amor a la vida se consigue con el paso del yoísmo a la filantropía, porque los educadores conseguimos nuestra plena gratificación abriéndonos hacia los educandos. Cuando nos entregamos sin importar las vicisitudes de la vida, estamos compartiéndonos en totalidad por el bien del otro. Gracias a esa donación, los maestros podemos amar y encontrar la verdad para actuar sabiamente en la vida escolar y personal. Con la entrega estamos compartiendo nuestra esencia, experiencias, virtudes y nuestra miseria, posibilitando una retroalimentación para potenciar habilidades y mejorar nuestro ser; gracias a esa interacción mutua, cotidiana entre docentes y estudiantes, la comunidad educativa logra ser justa, comprensiva, optimista, comprometida, colaboradora, motivada, reflexiva y amante de la vida.

Con el paso del yoísmo a la filantropía, la educación va mucho más allá del simple hecho de enseñar, a causa de la incidencia directa del docente en las personas. Hay que recordar que si los profesores no trabajamos por ser personas se nos dificultará formar personas. De manera que se hace ineludible que los profesionales de la educación realicemos el "arte regio" y "gastemos el tiempo" cultivando hábitos positivos, acordes a nuestra naturaleza, con el fin de transmitirlos a través del ejemplo y del currículo oculto. 
Así mismo, debemos conocernos a nosotros mismos, sufrir por amor, motivar y ser motivados, de esta manera la vida, dentro y fuera del escenario pedagógico, cobrará sentido en cuanto relacionemos lo personal-profesional y lo metal-emocional, y nos permitamos trascender el acto educativo hacia nuestra propia existencia. El vivir bien, entonces, requiere del desarrollo interior de cada docente y de la donación incondicional a quienes ofrecemos nuestro servicio, los educandos; es en ese momento cuando los profesionales de la educación seremos plenamente felices, amantes de la vida y libres.
Altet, M. (1993). La qualité des enseignants, seminairies d'enseignants. Raport final de l'etude francaise demsandée ar la Direction de l'Evaluation et de la Prospective. Nantes: Centre de recherches en eéducation.

Bauman, Z. (2011). Amor Líquido: acerca de la fragilidad de los vínculos humanos). México: Editorial Fondo de Cultura Económica.

Casanova, E. M. (1991). Las Ciencias de la Educación. Navarra: Librería del Seminario.

Day, C. (2006). Pasión por enseñar: La identidad personal y profesional del docente y sus valores. Madrid: Narcea S.A. Ediciones.

Despeyroux, D. (2013). El arte de vivir con filosofía. España: Océano Ámbar.

Diker, G., y Terigi, F. (1997). La formación de maestros y profesores: Hoja de ruta. Buenos Aires: Editorial Paidos.

Evans, L. (1997). Understanding Teacher Morale and Job Satisfaction. Teaching and Teacher Education, 31 (8), pp. 831-845.

García Cuadrado, J. A. (2010). Antropología Filosófica. Navarra: Ediciones Eunsa.

Hansen, D. T. (1999). Conceptions of Teaching and their Consequences. Louvain: Garant.

Isaacs, D. (2008). El trabajo de los profesores. Virtudes en los educadores: Justicia, comprensión y optimismo. Buenos Aires: Editorial EUNSA.

Lander, R. (1993). Repertories of Teaching Quality: A Contribution to the OECD/CERE Project, Teacher Quality from Case Studies of Six Swedish Comprehensive Schools. Goteborg: Department of Education and Science.

Llano, A. (2002). La vida lograda. España: Editorial Ariel.

Lorda, J. L. (2001). Moral, el arte de vivir. Madrid: Ediciones palabra.

Lyotard, J. (1979). The Postmodernalism and Condition: A report on Knowledge. Manchester: Manchester University Press.
Macconi, C. (1993). Teacher Quality Project: Italian Case Study. Roma: Ministero Pubblica Instruziones.

Melendo, T. (2002). Ocho lecciones sobre el amor humano. Madrid: Ediciones Rialph.

Ramsay, P. (1993). Teacher Quality: A case Study Prepared for the Ministry of Education as Part of the OECD Study on Teacher Quality. New Zeeland: Univerisity of Waikato.

Stork, Y., y Aranguren, J. (2003). Fundamentos de Antropología: Un ideal de excelencia humana. Navarra: Ediciones EUNSA.

Urbieta, J. R. (2006). El regalo de sí mismo: Educarnos para educar. Madrid: Narcea.

White, J. J., y Roesch, M. (2000). Listening to the Voices of Teachers: Examining Connections Between Student Performance, Quality of Teaching and Educational Policies in Seven Fairfax Country (VA) Elementary and Middle Public Schools. Maryland: University of Maryland. 